To appear in the proceedings of NATO ASI /Euroconference Formation and Interactions of Topological Defects, A. C. Davis and R. N. Brandenberger, eds. (Plenum, in press)
Los Alamos preprint

LAUR 95-170

Newton Institute

preprint NI 94044

\title{
COSMOLOGICAL EXPERIMENTS IN SUPERFLUIDS AND SUPERCONDUCTORS
}

\author{
W. H. Zurek \\ Theoretical Astrophysics, T-6, MS B288 \\ Los Alamos National Laboratory, Los Alamos, NM 87545
}

\begin{abstract}
Evolution of the order parameter in condensed matter analogues of cosmological phase transitions is discussed. It is shown that the density of the frozen-out topological defects is set by the competition between the quench rate - the rate at which the phase transition is taking place - and the relaxation rate of the order parameter. More specifically, the characteristic domain size which determines the typical distance separating topological defects in the new broken symmetry phase (and, therefore, their density) is determined by the correlation length at the instant at which the relaxation timescale of the order parameter is equal to the time from the phase transition. This scenario shares with the Kibble mechanism the idea that topological defects will appear "in between" domains with independently chosen broken symmetry vacuum. However, it differs from the original proposal in estimating the size of such domains through the non-equilibrium aspects of the transition (quench rate), rather than through the Ginzburg temperature at which thermally activated symmetry restoration can still occur in the correlation - length sized volumes of the broken symmetry phase. This scenario can be employed to analyze recent superfluid quench experiments carried out in bulk $\mathrm{He}^{4}$ to study the analogue of the "cosmological" prediction of significant vortex line production. It can be also applied to superfluid quenches in annular geometry, as well as to the rapid phase transition from the normal metal to superconductor, where the symmetry breaking occurs in the order parameter with the local (rather than a global) gauge. Cosmological implications of the revised defect formation scenario with the critical domain size set by the freeze-out time rather than by the Ginzburg temperature are also briefly considered.
\end{abstract}

\section{INTRODUCTION}

Expansion of the Universe following the Planck-era "Big Bang" inevitably leads to the decrease of temperature of the primordial fireball. This is thought to precipitate phase transitions which transform the vacuum from the "false" symmetric, 
high temperature phase to the low temperature broken symmetry "true" vacuum with the structure which defines "low energy physics" accessible to us in (high energy!) experiments. As the Universe undergoes phase transitions, the selection of the low temperature broken symmetry phase can only occur locally, within the causally correlated regions. It was noted by Zeldovich ${ }^{1}$ and his co-workers and especially by Kibble, ${ }^{2}$ that this symmetry breaking process may leave relics of the high energy phase - islands of the symmetric "false" vacuum - which will be trapped by the topologically stable configurations of the broken symmetry phase. Such topological defects would be massive and would therefore have observable consequences for the structures forming within the Universe as well as for the cosmic microwave background or for the evolution of the Universe as a whole.

Three principal kinds of topological defects ${ }^{3}$ are distinguished by their dimensionality. Monopoles are pointlike, and a disaster from the cosmological point of view. Membranes are two-dimensional, and (almost certainly) also a disaster: They would cause unacceptably large distortions of the cosmic microwave background. One - dimensional cosmic strings are by contrast a source of density perturbations which are still under investigation as a possible seed of structure formation.

Symmetry breaking phase transitions which occur in condensed matter physics are described by theories which are formally identical to those involved in the cosmological context, but have one crucial advantage: they can be studied in the laboratory. With this in mind, almost exactly a decade ago I have suggested ${ }^{4,5}$ that the cosmological mechanism for defect formation can be studied experimentally in the condensed matter context. The aim of this paper is to review this idea and to assess the experimental progress in implementing this cosmological scenario in the various condensed matter systems as well as to sketch possible directions for the future research.

This is an excellent time to undertake such a reassessment; the first realization of "cosmological experiments" has been accomplished a few years ago by Bernard Yurke and his colleagues in liquid crystals ${ }^{6,7}$. Even more recent exciting development is the experiment carried out by Peter McClintock and his colleagues ${ }^{8,9}$ who have implemented the original proposal by studying the superfluid transition in $\mathrm{He}^{4}$.

Liquid crystal experiments demonstrated that copious production of topological defects does indeed happen. ${ }^{6,7,10}$ But as the phase transition is of the first order, the interesting dynamics which takes place in the second order (Landau-Ginzburg like) phase transitions which are relevant to cosmology cannot be directly studied. The size of the characteristic domains (and the density of defects) is then set instead by the nucleation process. By contrast, liquid $\mathrm{He}^{4}$ becomes superfluid without nucleation. Therefore, while both liquid crystals and superfluids are of great interest, one might argue that the $\lambda$-transition into the superfluid allows one to address questions which cannot be posed in the liquid crystals context. (On the other hand, topological defects can be seen directly in liquid crystals, which means that the reverse of the above assertion is also true!) 
Both liquid crystals and superfluids are described by an order parameter with a global gauge symmetry. While global gauge field theories may be relevant to cosmological models, they seem to be an exception rather than the rule: Theories with local gauge symmetry are therefore even more interesting as an analogue of the cosmological phase transitions. Superconductors offer an obvious condensed matter example, and we shall also discuss the possibility of implementing "cosmological" quenches in this alternative low temperature setting.

Defect formation scenario in course of the rapid phase transitions is based on two assumptions. The first assumption is of the qualitative nature: It asserts that regions of the broken symmetry phase which are causally disconnected must select the new low temperature phase independently. As a result, when a symmetry-breaking phase transition with a non-trivial homotopy group occurs simultaneously in a sufficiently large volume, topological defects will appear with $\underline{\text { some }}$ density. The second assumption is of the quantitative nature: It involves specifying the process responsible for the causal propagation of "signals" which allows the choice of the new vacuum to occur in a coordinated (rather than independent) fashion. It leads to prediction of the density of topological defects.

Both assumptions are of course necessary ${ }^{2}$, but while the first one is straightforward and (with the benefit of hindsight) hard to argue with, the second one requires much more specific physical input. In the first order phase transitions the process which is responsible for the appearance of the new phase is nucleation: Small regions of the medium undergo thermal activation which takes them over the potential barrier separating "false" and "true" vacua. As a result, bubbles of a certain (critical) size appear and form seeds of the new phase. Eventually, through growth and coalescence of these bubbles new phase replaces the old one.

The original discussion of the scenario for defect formation appealed to a similar idea $^{2}$ : It was thought that thermally activated transitions between the correlationsized volumes of the new broken symmetry phase which are still possible well below the critical temperature $\mathrm{T}_{C}$ determine the initial density of the topological defects. Such transitions may occur down to the so-called Ginzburg temperature $\mathrm{T}_{G}\left(\mathrm{~T}_{G}<\right.$ $\mathrm{T}_{C}$ ), at which the (free) energy barrier becomes prohibitively large for correlationlength sized thermal fluctuations. If this were indeed the case, density of defects would be set by the correlation length at the Ginzburg temperature.

One of the key predictions of the original papers ${ }^{4,5}$ on "cosmological" phase transitions in superfluids was that this thermally activated process does not decide the density of defects: It was conjectured that the corresponding transitions are too local to result in "global" structures such as strings or domain walls. Instead it was proposed that the characteristic correlations length is set by the dynamics of the order parameter in the vicinity of the critical temperature $\mathrm{T}_{C}$.

Both in the superfluid (or, more generally, in the condensed matter) context and in the cosmological phase transitions selection of the new vacuum cannot be dynamically 
coordinated over regions larger than the size of the "relevant causal horizon." In superfluids (as well as in the case of other second-order phase transitions with a non-conserved order parameter) the velocity with which perturbations of the order parameter can propagate yields a natural "sonic horizon." I shall show that such considerations appear to lead to reasonable estimates of the density of topological defects in $\mathrm{He}^{4}$ superfluid quench experiments ${ }^{8,9}$, and demonstrate that this estimate differs from the one based on thermal activation and Ginzburg temperature.

The above "nonequilibrium" scenario ${ }^{4,5}$ represents a significant change of the point of view, and yields a prediction for the density of defects which is rather different from the "equilibrium" estimate based on the Ginzburg temperature. ${ }^{2}$ The aim of the rest of this paper is to physically motivate, describe, and investigate consequences of this "freeze-out" scenario on the example of superfluids. It is hoped that this discussion can be then generalized to the example of other phase transitions described by the non-conserved order parameter with global gauge symmetry. I shall also discuss the more complicated case of superconducting phase transition (where the broken symmetry phase is described by a locally gauge invariant theory) and comment on the implications of these considerations for the cosmological phase transition scenarios.

\section{SYMMETRY BREAKING IN SUPERFLUID HELIUM, SUPERCON- DUCTORS, AND IN THE EARLY UNIVERSE}

The aim of this section is to review some of the equilibrium properties of the systems which will serve as condensed matter analogues of the cosmological phase transitions. We shall carry out our discussion starting with the superfluid $\mathrm{He}^{4}$, go on to discuss superconductors, and finish with a brief overview of symmetry breaking in the field theories relevant for cosmological phase transitions. Many of the prospective readers of this paper may be used to the order - common in the cosmology/particle astrophysics presentations - which covers the same ground, but in the opposite directions, and with a complementary emphasis. I have adopted this order starting with the low temperature phase transitions for several reasons: to begin with, it is meant to emphasize that the most accessible testing grounds for the ideas we shall be considering are in the simplest low temperature systems. (This belief has been strengthened by the recent experiments of McClintock and his co-workers. ${ }^{8,9}$ ) Moreover, this sequence reflects the original flow of ideas (where the condensed matter analogues were used to elucidate spontaneous symmetry breaking in the field-theoretic context). Finally, this order correlates with the degree of confidence we can have also in the non-equilibrium aspects of various examples of the phase transitions, and especially in the resulting scenarios of topological defect formation.

\section{Superfluid Helium}

Superfluid forms in the low temperature $\left(\mathrm{T}<2.18^{\circ} \mathrm{K}\right)$, moderate pressure $(\mathrm{p}<25$ 
atm) corner of the $\mathrm{He}^{4}$ phase diagram. Various manifestations of superfluidity (like flow with negligible friction, persistent currents, etc.) are well-documented in the literature. ${ }^{11}$ They can be accommodated and explained the context of several phenomenological models, which tend to emphasize various properties of superfluid helium. Thus, two-fluid model introduced by Tisza regards superfluid $\mathrm{He}^{4}$ as a mixture of two components - superfluid and normal. The fraction of the fluid which is either "normal" or "super" depends on the distance from the (pressure dependent) temperature $\mathrm{T}_{\lambda}$ at which transition to the normal $\mathrm{He}^{4}$ liquid occurs, which is known as the " $\lambda$ line" (because of the asymmetric form of the specific heat near $\mathrm{T}_{\lambda}$ ).

While normal $\mathrm{He}^{4}$ is simply another liquified noble gas, properties of the superfluid fraction can be qualitatively understood when it is regarded as a Bose condensate of $\mathrm{He}^{4}$ atoms. In particular, in addition to the normal "first sound" (in which density perturbations propagate as in the air) superfluid $\mathrm{He}^{4}$ can carry (albeit much more slowly) the second sound (where the relative densities of the super and normal components are perturbed, but in such a way that the total density remains constant).

Phonons of the second sound are not the only new excitations in the superfluid phase: Rotons - massive excitations - also appear below $\mathrm{T}_{\lambda}$. Appearance of a new massless and a new massive "particle" in the broken symmetry phase below $\mathrm{T}_{\lambda}$ is strongly reminiscent of the Goldstone boson and of the Higgs particle which should accompany breaking of the global symmetry during phase transitions.

To pursue this (imperfect, as it turns out) analogy further we shall rely on the Landau-Ginzburg theory of the second order phase transitions. There, the specific free energy of the system is given in the vicinity of the phase transition by the analytic expression of the form:

$$
F(\Psi)=\frac{\hbar^{2}}{2 m}|\vec{\nabla} \Psi|^{2}+\alpha|\Psi|^{2}+\frac{\beta}{2}|\Psi|^{4} .
$$

Here $\Psi$ is the space-dependent order parameter, an abstract measure of the degree to which the symmetry in question has been broken, while $\alpha$ and $\beta$ are parameters:

$$
\begin{gathered}
\alpha=\alpha^{\prime}\left(T-T_{C}\right) / T_{C}, \quad \alpha^{\prime}>0 ; \\
\beta=\mathrm{const}>0 .
\end{gathered}
$$

In addition to the two "potential" terms (which depend on the even powers of the order parameter), Eq. (1) contains the square of the gradient, the "kinetic energy" contribution to the free energy. The mass $m$ is usually taken to be that of the $\mathrm{He}^{4}$ atom, but is in fact a parameter which is fixed by the normalization so that $|\Psi(\vec{r})|^{2}$ yields the correct density of the superfluid.

As the temperature drops below the critical $T_{C}=T_{\lambda}$, the shape of the potential contribution to the free energy changes. Instead of a single minimum in a disordered phase with $\Psi=0$ one now expects the field to have a typical amplitude given by:

$$
\sigma=\sqrt{-\alpha / \beta},
$$


and a random phase.

In the application of Landau-Ginzburg theory to superfluid $\mathrm{He}^{4}$ one tends to regard the order parameter $\Psi$ as a wave function of the Bose condensate. $\Psi$ is then a complex field, and its instantaneous configurations need to be characterized by both its amplitude and its phase as a function of position. The simplest such solution is of the form:

$$
\Psi=\sigma \exp (i \theta)
$$

where $\theta$ is constant.

To investigate more interesting (and more complicated) solutions it is useful to rescale the free energy of Eq. (1) in terms of $\sigma$ and of the correlation length:

$$
\xi=\frac{\hbar}{\sqrt{2 m|\alpha|}}
$$

In superfluid $\mathrm{He}^{4}$, well below $\mathrm{T}_{C}$, the correlation length is of the order of a few Angstroms. In terms of the new variables $\varrho=r / \xi, \eta=\Psi / \sigma$ the condition for the stable configuration of $\Psi$ (i.e., for a minimum of $F(\Psi)$ ) can be expressed as:

$$
\nabla^{2} \eta=\left(|\eta|^{2}-1\right) \eta
$$

In addition to the trivial solution given by Eq. (4) (i.e. $|\eta|=1$ ) Eq. (6) also admits axisymmetric solutions of the form:

$$
\eta=\psi(\varrho) \exp i n \phi
$$

where $(\varrho, \phi, z)$ are the cylindrical coordinates. Here, $n$ must be a natural number (otherwise, $\eta$ would not be single-valued). The radial part of the physically relevant solution is regular near the origin $\left(|\psi| \sim \varrho^{n}, \varrho \ll 1\right)$ and approaches equilibrium density at large distances $\left(|\psi|^{2} \simeq 1-n^{2} / \varrho^{2}, \varrho \gg 1\right)$. The phase of the complete solution is $\theta=n \phi$ on any $\varrho=$ const $>0$ circle, but remains undefined along the singular $\varrho=0$.

Since $\Psi$ is the wave function, the gradient of the phase gives the local superfluid velocity;

$$
\overrightarrow{v_{S}}=\frac{\hbar}{m} \vec{\nabla} \theta(\vec{r})
$$

where $m$ is the mass of the $\mathrm{He}^{4}$ atom. Therefore, the axially symmetric solution of Eq. (6) is a vortex line with a core of width given by the correlation length $\xi$, Eq. (5). The superfluid circulates with a radius-dependent velocity given by:

$$
\left|\overrightarrow{v_{S}}\right|=v_{\varphi}=\frac{\hbar}{m} \frac{n}{r}
$$


Inside the core a symmetric vacuum - the normal fluid - makes up for the density deficit caused by the decrease of $|\Psi|^{2}$. $n$ is known as a winding number. For energetic reasons vortex lines with $n>1$ tend to dissolve into vortices with $n=1$.

Existence of the vortex lines in the superfluid Helium 4 has been postulated by Onsager and Feynman as the only means of introducing rotation into the superfluid without violating the condition of the single-valuedness of the Bose condensate wavefunction $\Psi$. Their existence has been since confirmed and their properties were carefully studied $^{11}$. Seen from the vantage point of the Landau-Ginzburg theory of superfluidity vortex lines are a perfect example of a global topological defect ${ }^{3}$.

The analog of the vortex line in field theories relevant in the cosmological context is a cosmic string. As it was noted by Kibble, ${ }^{2}$ strings form when the first homotopy group $\Pi_{1}(G / H)$ - where $G$ and $H$ are the symmetry groups before and after the phase transition - is nontrivial. For superfluid helium this is indeed the case, as $G / H=U(1)$, and $\Pi_{1}(G / H)=Z$. Superfluid vortex line is an analogue of a global string - that is, a string associated with the breaking of a global gauge symmetry. The alternative local strings are like the flux lines in superconductors. We shall consider them below.

\section{Superconductors}

Landau-Ginzburg model of the second order phase transition is only a qualitative approximation for the superfluids, but it turns out to be a quantitatively accurate mean field theory for superconductors ${ }^{12}$. It is based on the observation that the wave function of the Bose condensate of Cooper pairs - which is the relevant order parameter - has a free energy density given by:

$$
F=\frac{1}{4 m}\left|\left(-i \hbar \vec{\nabla}-\frac{2 e}{c} \vec{A}\right) \Psi\right|^{2}+\alpha|\Psi|^{2}+\frac{1}{2} \beta|\Psi|^{4}+\frac{B^{2}}{8 \pi}+E_{0}
$$

where $2 m$ and $2 e$ are a mass and a charge of a Cooper pair, and where we have incorporated terms due to external magnetic field in the constant $E_{0}$. Equation (10) differs from the Eq. (1) through the presence of electromagnetic (gauge) fields - vector potential $\vec{A}$ enters into the kinetic term through the usual replacement;

$$
\vec{\nabla} \longrightarrow \vec{\nabla} \pm \frac{2 i e}{\hbar c} \vec{A}
$$

and the induction $\vec{B}$ is given by:

$$
\vec{B}=\vec{\nabla} \times \vec{A}
$$

Symmetry breaking occurs below the phase transition temperature $\mathrm{T}_{C}$ when the coefficient $\alpha(T)=\alpha^{\prime}\left(T-T_{C}\right) / T_{C}$ becomes negative. As in the superfluid $\mathrm{He}^{4}$ the 
order parameter acquires a finite vacuum expectation value, Eq. (3), and an associated phase $\theta$.

Quantized vortices in superconductors emerge in a manner analogous to the vortex lines in superfluid. To see this, let us consider a closed loop in the real space. Suppose that the phase of the broken symmetry vacuum changes by $2 \pi$ as one follows the path along the loop. In the superconductor, the current is related to the gradient of the phase through:

$$
\vec{J}=2 e|\Psi|^{2} \vec{v}_{S}
$$

where the velocity of the Cooper pairs is given by:

$$
\hbar \vec{\nabla} \theta=2 m \overrightarrow{v_{S}}+2 e \vec{A} / c .
$$

With a few additional assumptions about the axisymmetry of the solution one can then employ Eq. (13) to calculate magnetic induction:

$$
B=\frac{\Phi_{0}}{2 \pi \lambda^{2}} K_{0}(r / \lambda)
$$

Here $K_{0}$ is the zero - order Hankel function of imaginary argument and $\Phi_{0}$ is the flux quantum;

$$
\Phi_{0}=h c / 2 e,
$$

where $c$ is the speed of light, and equals to $2.07 \cdot 10^{-7}$ gauss $\mathrm{cm}^{2}$.

London penetration depth $\lambda$ is given by

$$
\lambda^{2}=m c^{2} /\left(8 \pi e^{2} n_{C}\right)
$$

with the equilibrium density of Cooper pairs:

$$
n_{C}=\sigma^{2}=|\alpha| / \beta
$$

Thus, the flow pattern, and with it $B$, die of exponentially on a scale set by $\lambda$. This scale on which electromagnetic interactions fall off exponentially can be either small or large compared to the correlation length of the order parameter in superconductors:

$$
\xi^{2}=\hbar /(4 m \alpha)
$$

which is analogous to the superfluid correlation length, Eq. (5). The value of $\xi$ determines the size of the core of a vortex - size of the region where there is no Bose condensate of Cooper pairs.

In a typical superconductor far below $\mathrm{T}_{C}$ correlation length has values of the order of $\xi_{0} \cong 10^{3} \AA$, two orders of magnitude larger than the corresponding quantity in the superfluid. Thus while in the superfluid correlation length is of the order of the interatomic spacing, in the superconductors it is at least two orders of magnitude 
larger. This is the main reason why the mean field Landau-Ginzburg theory is quite accurate for superconductor, but only qualitatively correct for superfluids. ${ }^{11}$

The ratio of the penetration depth and of the correlation length does not depend on temperature and is the fundamental parameter of the theory, crucial for the existence of vortices. This is because the flow around the vortex has an inner radius given by $\xi$ and an outer radius given by $\lambda$. Thus, $\xi$ must be smaller than $\lambda$ if the vortex lines are to exist. The exact condition turns out to be:

$$
\kappa=\lambda / \xi>1 / \sqrt{2}
$$

This is the condition which distinguishes between type I superconductors, in which vortices are not found, and type II superconductors, satisfying inequality (20), in which they can exist.

Superfluid vortex was an analogue of a global string. Superconducting vortices are analogous to local strings - the solution I have briefly sketched out above is similar to the Nielsen-Olesen string solution in the field theories with local gauge.

\section{Field Theory}

Expressions (1) and (10) for the free energy of a superfluid or of a superconductor have more general field-theoretic analogs. Thus, one can consider a complex field $\varphi$ with a Lagrangian (we adopt in this section convenient units $\hbar=c=1$ ) given by:

$$
L(\varphi)=\left(\partial_{\mu} \varphi^{*}\right)\left(\partial^{\mu} \varphi\right)-\alpha \varphi^{*} \varphi-\frac{\beta}{2}\left(\varphi^{*} \varphi\right)^{2}
$$

For $\alpha<0, \beta>0$, the potential in (21) has a minimum when the absolute value of $\varphi$ is given by $\sigma=\sqrt{-\alpha / \beta}$ of Eq. (3). In addition to the topological defects (which can be shown to exist through a discussion analogous to our above derivation of vortex lines in superfluids), Eq. (21) can be used to demonstrate existence of massive and massless modes in the broken symmetry vacuum. To show this, one considers small perturbations around the broken symmetry ground state (which can be set for the purpose of this calculation to be completely real):

$$
\varphi(x)=\sigma+(u(x)+i v(x)) / \sqrt{2} .
$$

Substituting this into Eq. (21) and ignoring constant terms one recovers:

$$
L=\frac{1}{2}\left(\partial_{\mu} u\right)^{2}+\frac{1}{2}\left(\partial_{\mu} v\right)^{2}-\beta \sigma^{2} u^{2}-\frac{\beta}{\sqrt{2}} \sigma u\left(u^{2}+v^{2}\right)-\frac{\beta}{8}\left(u^{2}+v^{2}\right)^{2}
$$

We regard Eq. (23) as a Lagrangian for the coupled fields, $u$ and $v$. It implies that the field $u$ which varies the amplitude of $\varphi$ has a positive mass (given by $\beta \sigma^{2}=-\alpha=|\alpha|$ ), 
while the variations of $v$ (phase) are massless. These massless excitations correspond to Goldstone bosons.

By analogy with the field theoretic considerations, one would therefore expect existence of two modes of excitations in the broken symmetry phase of systems described by a complex, non-conserved order parameter such as the superfluid helium. Two such modes - second sound phonons and rotons - do indeed appear, but their correspondence to the Goldstone bosons and massive excitations is at best imperfect. We shall not pursue this aspect of the analogy further in our discussion: In absence of a detailed theory of superfluid $\mathrm{He}^{4}$ it is hard to carry out such an investigation with a satisfactory degree of rigor.

Let us now consider the case of a local gauge theory. The corresponding Lagrangian is:

$$
L=-\frac{1}{4} B_{\mu \nu} B^{\mu \nu}+\left[\left(\partial_{\mu}+i e A_{\mu}\right) \varphi^{*}\right]\left[\left(\partial_{\mu}-i e A_{\mu}\right) \varphi\right]-\alpha \varphi^{*} \varphi-\frac{\beta}{2}\left(\varphi^{*} \varphi\right)^{2}
$$

where $A_{\mu}$ is a massless gauge boson, and $B_{\mu \nu}=\partial_{\mu} A_{\nu}-\partial_{\nu} A_{\mu}$. In contrast to the Lagrangian (21) which was invariant under global gauge transformations, Eq. (24) is invariant under the local Abelian gauge transformation;

$$
U(\theta(x))=e^{-i \theta(x)}
$$

where $\varphi(x) \longrightarrow e^{-i \theta(x)} \varphi(x)$, and $A_{\mu}(x) \longrightarrow A_{\mu}(x)-\frac{1}{e} \partial_{\mu} \theta(x)$.

When we carry out an expansion around the local minimum of the potential for the case $\alpha<0$ we have considered previously, we find:

$$
L=-\frac{1}{4} B_{\mu \nu} B^{\mu \nu}+e^{2} \sigma^{2} A_{\mu} A^{\mu}+\frac{1}{2}\left(\partial_{\mu} u\right)^{2}+\frac{1}{2}\left(\partial_{\mu} v\right)^{2}-\beta \sigma^{2} u^{2}-\sqrt{2} e \sigma A_{\mu} \partial_{\mu} v+\ldots
$$

The term involving $A_{\mu} A^{\mu}$ is the surprising outcome of the symmetry breaking - it looks as if the gauge vector field has acquired a mass.

The Lagrangian (26) can be further simplified by fixing the gauge so that $\theta(x)$ is equal to the phase of the original complex field $\varphi(x)$. In this gauge:

$$
\varphi(x)=\sigma+u(x) / \sqrt{2}
$$

and the Lagrangian becomes:

$$
L=-\frac{1}{4} B_{\mu \nu}^{\prime} B^{\prime \mu \nu}+e^{2} \sigma^{2} A_{\mu}^{\prime} A^{\prime \mu}+\frac{1}{2}\left(\partial_{\mu} u\right)^{2}-\left(\beta \sigma^{2}\right) u^{2}-\frac{1}{8} \beta u^{4}+\frac{1}{2} e^{2}\left(A_{\mu}^{\prime}\right)^{2}\left(\sqrt{2} \sigma u+u^{2}\right) .
$$

In this form it is apparent that $L$ describes interaction of the massive vector boson $A_{\mu}^{\prime}$ with a real scalar field $u$, (the "Higgs boson") with the mass squared given by a $\beta \sigma^{2}=-\alpha$. 


\section{Summary}

The analogy between symmetry breaking in superfluid helium and in the field theories with global gauge invariance is now apparent: Wavefunction of the Bose condensate is the analog of the scalar field. Static global cosmic strings and vortex lines in He II are a solution to an identical time-independent equation of a form:

$$
\nabla^{2} \varphi \sim-\alpha \varphi+\beta|\varphi|^{2} \varphi
$$

Their structure is defined by the correlation length $\xi$, which sets the size of the core of the vortex. The total "string tension" is associated with the kinetic energy of circulation, and is (in both cases) logrithmically dependent on the cutoff at large scales.

Analogy in the case of the local gauge is even more striking. There the scalar field corresponds to the Bose condensate of Cooper pairs, and the gauge field which acquires mass in the broken symmetry phase is analogous to electromagnetic (gauge) field which becomes massive in the superconducting state. There are now two characteristic lengths - the correlation length $\xi$ of the scalar Bose condensate and the penetration depth $\lambda$. The analogy between the superconducting vortex and the Nielsen-Olesen string was already pointed out.

\section{FREEZE-OUT OF TOPOLOGICAL DEFECTS IN RAPID PHASE TRANSITIONS}

In the preceding section, we have focused on the analogies between the static properties of the broken symmetry phase. In particular, we have identified topologically stable time-independent solutions in both superfluids and in superconductors. Here we shall quantify the general considerations of the first, introductory section, and derive the density of vortex lines in superfluid $\mathrm{He}^{4}$ which can be obtained throughout the rapid (pressure) quench. This experiment was originally suggested ${ }^{4,5,13}$ for the $\lambda$-line transition into the superfluid He II, but its analogs can be carried out in

other phase transitions. Slightly different (because of the first order nature of the phase transition) case of liquid crystals was studied experimentally by Yurke and his collaborators $^{6,7}$ and by Bowick and his colleagues ${ }^{10}$.

The case of $\mathrm{He}^{4}$ has been implemented more recently by McClintock and his colleagues $^{8,9}$. It will be the focus of this section. We shall review the "freeze-out" scenario, argue that Ginzburg temperature does not play the key role in determining the initial density of defects, estimate vortex line density from the freeze-out argu-

ment, and show that it is consistent with the experiment. We shall also consider the case of superconductors.

\section{Quench into Superfluid}

The transition from normal to superfluid $\mathrm{He}^{4}$ is a particularly suitable (from the 
experimental point of view) analog of the cosmological phase transitions. In addition to the parallel between the Bose condensate and scalar fields we have discussed in the last section, pressure quench through the $\lambda$-line offers quick (dynamical) method of reaching the state with the broken global gauge symmetry ${ }^{4,5,13}$. Moreover, the speed of the first sound (which will limit the rate at which change of the pressure will be communicated through the medium) is - near the superfluid phase transition $\mathrm{T}_{\lambda}$ - orders of magnitude in excess of the second sound velocity, which limits the speed with which perturbations of the Bose condensate can spread. Thus, we can reproduce in the superfluid phase transition the "acausal" nature of the cosmological phase transitions - dynamics of the broken symmetry phase is slow compared to the dynamics of the quench.

Before the phase transition we imagine the order parameter which can locally (i.e. within the $\xi$-sized region) fluctuate, but which is on the average in the symmetric state. This initial state will change on the characteristic relaxation timescale:

$$
\tau=\hbar /|\alpha|
$$

This timescale will be essential in our considerations. We shall use it to estimate the expected density of vortex lines in the superfluid through the following freezeout scenario: We note that as the pressure drops, the dimensionless temperature parameter:

$$
\epsilon(T)=\left(T-T_{\lambda}\right) / T_{\lambda}
$$

which is positive in the normal He I, decreases and becomes negative. When $T$ is close to $T_{\lambda}$ relaxation time becomes very large, and it blows up to infinity on the $\lambda$-line;

$$
\tau=\tau_{0} /|\epsilon|
$$

Thus, the order parameter (which changes on the timescale given by $\tau$ ) will be able to adjust only very slowly to the changes of thermodynamic parameters. This critical slowing down is accompanied by an increase of the correlation length $\xi$, which diverges as:

$$
\xi=\xi_{0}|\epsilon|^{-\nu}
$$

in the vicinity of $\epsilon=0$. Landau-Ginzburg theory with $\nu=\frac{1}{2}$, and $\xi_{0}=5.6 \AA$ provides an acceptable (although not an optimal, as we shall see shortly) fit for superfluid helium.

In course of a quench we shall imagine that - very near the $\lambda$-line - dimensionless temperature will be approximately proportional to the time before (after) $T=T_{\lambda}$ is reached:

$$
\epsilon=t / \tau_{Q}
$$

Here $\tau_{Q}$ is the quench timescale.

When $\tau \ll t$ the order parameter reacts to the quench-induced change of $\epsilon$ by adjusting its average state (i. e., by increasing the correlation length) in an essentially 
adiabatic fashion: For each new value of $\epsilon$ there is a new realization of a nearequilibrium with the approximately adjusted correlation length, average size of $|\Psi|^{2}$, etc. However, as $T$ approaches $T_{\lambda}, \epsilon$ decreases, and the relaxation timescale becomes larger and larger.

At some point the rate at which the thermodynamic changes occur will become greater than the rate with which the order parameter $\Psi$ can adjust. At that instant our "adiabatic" approximation will cease to be sufficient: Very close to $T_{\lambda}$ the configuration of the order parameter will be essentially "frozen out" by the sluggishness of its dynamics. "Impulse" approximation will now apply: order parameter will not be able to adjust - the correlation length will not be able to increase so as to keep up with its equilibrium value prescribed by Eq. (32).

The time $\hat{t}$ when the crossover from the "adiabatic" to the "impulse" regime occurs is of course critically important: It will determine the size of the "frozen out" domains. We can compute $\hat{t}$ by equating the time-dependent relaxation time with the time from the phase transition;

$$
\tau(\hat{t})=\hat{t}
$$

or;

$$
\tau_{0} /\left(\hat{t} / \tau_{Q}\right)=\hat{t}
$$

which finally yields:

$$
\hat{t}=\sqrt{\tau_{0} \tau_{Q}} .
$$

We are now nearly done. To obtain the initial density of the quench-generated vortex lines we still need to compute the characteristic correlation length set by the "freeze out" at $t=\hat{t}$ (or for $\epsilon=\epsilon(\hat{t})$ ). This yields:

$$
d=\xi(\hat{t})=\xi_{0}\left(\tau_{Q} / \tau_{0}\right)^{\nu / 2} .
$$

In the Landau-Ginzburg theory $\nu=\frac{1}{2}$. The corresponding vortex line density is then given by:

$$
\ell=k / d^{2}
$$

where $k$ is a proportionality constant which is thought to be smaller then, but probably of the order of 1 .

Equation (39) can be further rewritten to yield density of the vortex lines in terms of the quench timescale:

$$
\ell=\left(k / \xi_{0}^{2}\right) \cdot\left(\tau_{0} / \tau_{Q}\right)^{\nu} .
$$

The exponent $\nu=\frac{1}{2}$ in the Landau-Ginzburg theory, but as we have already mentioned, Landau-Ginzburg theory is only qualitatively correct for superfluid helium. Renormalization group prediction for $\nu$ is;

$$
\nu=\frac{2}{3}
$$


Experiments seem to agree with Eq. (41). Thus, for example the correlation length determined from measurements is very well fit by:

$$
\xi(\epsilon)=\xi_{0} /|\epsilon|^{0.67}
$$

where $\xi_{0}=4 \AA$. Similarly, the velocity of the second sound is approximately given by:

$$
u=u_{0}|\epsilon|^{1-\nu},
$$

where $u_{0} \cong 47 \mathrm{~m} / \mathrm{s}$ which is suitable for the renormalization group exponent $\nu=\frac{2}{3}$ yields a better fit than the still acceptable $u_{0} \cong 70 \mathrm{~m} / \mathrm{s}, \nu=\frac{1}{2}$ which obtains from the Landau-Ginzburg theory.

\section{Ginzburg Temperature and the Thermal Activation of Defects}

For contrast, let us consider the activation mechanism for the formation of topological defects. The idea is quite straightforward: For some range of temperatures below the phase transition temperature $T_{C}=T_{\lambda}$ thermal fluctuation will be able to "flip" the order parameter in the system between the local minima around the rim of the "Mexican hat" potential. Regions which will undergo such transitions will have sizes of the order of the correlation length $\xi$. Therefore, the specific free energy barrier computed from Eq. (1) is:

$$
F(0)-F(\sigma)=\alpha^{2} /(2 \beta)
$$

The energy of a volume of the size of a correlation length will be comparable to the available thermal energy when:

$$
\xi^{3} \alpha^{2} / 2 \beta \cong k_{B} T
$$

Since $\xi \cong \hbar / \sqrt{2 m|\alpha|}$, the left hand side of the above equation varies as $\left(T-T_{C}\right)^{1 / 2}$. A numerical estimate for the Ginzburg temperature $T_{G}$ for which Eq. (45) is satisfied yields;

$$
T_{\lambda}-T_{G} \cong 0.5\left[{ }^{\circ} K\right]
$$

for superfluid Helium II. This estimate is in reasonable agreement with the width of the so-called $\lambda$ anomaly in the specific heat (the shape of which is responsible for the name " $\lambda$-line" of the transition from normal to super phase of liquid helium), but it seems to have little bearing on the existence or stability of vortex lines. We shall confirm this immediately below, while comparing theoretical prediction of vortex line production in a quench with experimental results. Indeed, this conclusion seems sensible and valid for superfluids even without appealing to the recent experiments: For, if small scale (correlation length $\xi$ ) fluctuations between the degenerate minima of the potential could create topological defects, then this process would also destroy them on the timescale given by $\tau$. This is known not to happen to vortex line density 
generated by various methods in a superfluid above the Ginzburg temperature $T_{G}$ (but, of course, below $T_{\lambda}$ ).

The reason $T_{G}$ does not play a decisive role in creation of vortex lines in $\mathrm{He}^{4}$ (and, for that matter, should not be an important mechanism for production of other topological defects in quench - induced phase transitions either in condensed matter or in cosmology) is likely to be associated with the spatial extent of the thermally activated transitions: Local thermal fluctuation can perhaps create small loops of vortex line, but these loops will have a radius approximately equal to the size of their core (since both are defined by the same correlation length $\xi$ ). Such ill-defined vortex line "doughnuts" are unlikely to be stable - after all, they represent a configurations which may be a shallow local minimum of the free energy, but which have a higher free energy then the uniform superfluid. Hence, they are not large enough to be really topologically stable - change of the field configuration in a finite region of space the order of $\xi$ suffices to return to the uniform "true vacuum". (Indeed, one is tempted to speculate that the rotons - which become plentiful above $T_{G}$ - are thermally excited in such fashion).

It is quite evident that such local loops cannot result in creation (or destruction) of one long vortex line which is likely to be the dominant contribution to the vortex line density following pressure quench. Long lines or large loops created by the freezeout can become "wrinkled" as a result of thermal activation on the Ginzburg scale, but this is not expected to lead to a significant increase of $\ell$, Eq. (40). Similar reasoning can be repeated for membranes. Such requirements are least convincing in the case of monopoles (which have spatial extent of order $\xi$ in every direction). However even monopoles will have to be created "in pairs" by thermal fluctuations. These pairs of monopoles of opposite charge will be separated by distance of order $\xi$. Therefore, they are not likely to separate and survive.

We are led to the conclusion that the dominant process in creation of topological defects will have to do with the critical slowing down and a consequent "freeze-out" of the fluctuations of the order parameter at the time $\hat{t}$ rather than with the thermal activation and Ginzburg temperature. We shall evaluate this conclusion in the light of experimental results below.

\section{Comparison with the Experiment}

The experiment carried out at the Lancaster University ${ }^{8,9}$ follows the pressure quench strategy proposed for the superfluid helium one decade ago. ${ }^{4,5,13}$ The typical $\Delta \epsilon$ - change of the relative temperature - can be crudely estimated from the initial and final temperature differences:

$$
\Delta \epsilon \cdot T_{\lambda} \simeq\left[T_{i}-T_{\lambda}\left(p_{i}\right)\right]-\left[T_{f}-T_{\lambda}\left(p_{f}\right)\right]
$$

where $T_{i}, p_{i}$ and $T_{f}, p_{f}$ are the initial and final temperature and pressure respectively. This method yields $\Delta \epsilon \sim 0.1$, which, combined with the time interval $\Delta t \sim 3 \mathrm{~ms}$ over 
which pressure drop occurs ${ }^{8}$, results in the estimate of the quench timescale:

$$
\tau_{Q} \simeq \Delta t / \Delta \epsilon \simeq 30 \mathrm{~ms}
$$

I would like to emphasize that this is a very rough estimate of the actual $\tau_{Q}$. To obtain a more reliable $\tau_{Q}$ one would have to compute quench rate along the isentrope ( $S=$ const) of the quench:

$$
\left(\tau_{Q}\right)^{-1}=(\partial \epsilon(t) / \partial t)_{S=\text { const }, \epsilon=0}
$$

for the point at which the phase transition occurs (that is, for $\epsilon=0$ ). I have not carried out such a calculation, but the shapes of the isentropes near $T_{\lambda}$ lead one to believe that $\tau_{Q}$ given by Eq. (49) would be somewhat (perhaps even by an order of magnitude or so) larger than the simpler estimate of Eq. (48) would have it.

With these estimates (and the associated caveats) in mind let us now use Eq. (40) to estimate initial vortex line densities. For the Landau-Ginzburg theory $\left(\nu=\frac{1}{2}\right.$ with $\xi_{0}=5.6 \AA$ and $\tau_{0}=0.85 \cdot 10^{-11} \mathrm{~s}$ ) one obtains a prediction:

$$
\ell_{L G} \cong 3\left(\tau_{Q} / 100 \mathrm{~ms}\right)^{-1 / 2} \cdot 10^{13} \mathrm{~m}^{-2}
$$

while for renormalization group theory the prediction is:

$$
\ell_{R G} \cong 1.2\left(\tau_{Q} / 100 \mathrm{~ms}\right)^{-2 / 3} \cdot 10^{12} \mathrm{~m}^{-2}
$$

These predictions bracket the lower bound of $\ell \sim 10^{13} \mathrm{~m}^{-2}$ based on the experimental results. Vortex line production occurs also where a quench is initiated just below $(\sim 10 \mathrm{mK})$ the $\lambda$-line. There is, however, no noticeable vorticity produced in quenches which start far below the phase transition. This is an intriguing observation. A possible explanation of this effect is to appeal to the combination of the flows which are also induced by the quench ${ }^{9}$ and the thermally activated vortex line production. What can happen is that rare, thermally excited vortex lines get stretched and entangled by the flows generated in course of the expansion of the $\mathrm{He}^{4}$ container. ${ }^{9}$ This may result in a turbulent tangle of vortex lines, amplifying the pre-existing thermally activated "seed" vorticity. If this mechanism does indeed operate, it is likely that flows also amplify the density of vortex lines in quenches which cross $T_{\lambda}$.

There are also serious concerns about the accuracy of the estimate derived from the "naive" application of the freezeout scenario to superfluid $\mathrm{He}^{4}$. Thus, for example, the estimated "freeze out" correlation length (Eq. (38)) is only an order of magnitude estimate of $d$. Indeed, it seems likely that the actual correlation length will be bounded from above by the freeze out scale, but could be somewhat (by a factor of a few) smaller than $\xi(\hat{t})$, Eq. (38). Moreover, an accurate estimate of $k$ of Eq. (40) would be useful. Last but not least, the very process of formation of the Bose condensate is unlikely to be instantaneous, and - as it was already pointed out ${ }^{13}$ - large vortex line densities may depress $T_{\lambda}$. 
In spite of these reservations, the obvious conclusion of this section is that the rapid quench generates vortex line density consistent with the theoretical predictions based on the idea of the "freeze out" of the configurations of the order parameter." Moreover, it is also clear that the Ginzburg temperature does not play as decisive a role as it was originally expected ${ }^{2}$.

\section{Quench into the Superconducting State}

Much of what we can anticipate in the case of a quench into a superconductor will be based on an argument which parallels the case of superfluid $\mathrm{He}^{4}$. We shall therefore be brief in the discussion of vortex line creation in (type II) superconductors. In effect, we shall repeat what was already said before, but we shall also emphasize the differences between the two cases.

It should be noted that some of these differences are non-trivial. Thus, presence of the gauge field in superconductors complicates the problem by adding extra physics which leads, for example, to an additional characteristic scale. On the other hand, superconductors are rather well described by the simple Landau-Ginzburg theory with the order parameter representing the wavefunction of the Bose condensate of Cooper pairs. $^{12}$

Let us begin by noting that, in contrast to just one superfluid $\mathrm{He}^{4}$, the list of superconducting materials is very long (even if we restrict ourselves to type II only). This is especially true if one includes in it new "high $T_{C}$ " superconductors. In general, temperature of the phase transition into the superconducting state is not very sensitive to pressure. ${ }^{14}$ Nevertheless, some sensitivity to pressure does exist (especially in the high- $T_{C}$ materials) so one could contemplate a similar "quench" scenario as the one described in the case of $\mathrm{He}^{4}$. This would have the advantage of achieving the transition very quickly and in a manner which does not directly involve electrons. However, if the pressure quench proved to be impossible, cooling might be an interesting alternative, and could be achieved relatively rapidly when the sample is a thin layer of superconducting material.

This 2-D (rather than truly 3-D) strategy may have one more advantage: Vortex lines which form inside the superconductor cannot be really "seen" from the outside (in superfluid helium second sound attenuation can be used to measure vortex line density). And since superconducting vortices in the bulk are invisible, one may as well concentrate on the points where they enter or leave the sample, which suggests very flattened samples.

Two-dimensional geometry may also help address one more likely problem: Vortex lines created in the superconductor will have a tendency to annihilate or to escape from the sample (after all, the state without topological defects and without the associated magnetic fields has lower energy!). Using flattened samples may allow one to trap (pin) vortex lines (and thus slow down annihilation).

With all these caveats in mind, let us now estimate the density of vortex line 
generated in a rapid quench in the type II superconductor. Proceeding along the path parallel to the one we have adopted for the superfluid phase transition, we are led to evaluate the relaxation timescale of the order parameter $\tau$ for superconductors; $\tau=\tau_{0} /|\epsilon|$ (see Eq. (32)) where $\tau_{0}$ can be approximately computed from the so-called Gorkov equation to be:

$$
\tau_{0}=\frac{\pi \hbar}{16 k_{B} T_{C}} .
$$

The freeze out time will be still given by $\hat{t}=\sqrt{\tau_{0} \tau_{Q}}$, Eq. (37), which with the help of Eq. (51) can be numerically evaluated to be:

$$
\hat{t} \cong 1.225 \cdot \sqrt{\tau_{Q} / T_{C}}[\mu s]
$$

when the quench timescale $\tau_{Q}$ is in seconds and $T_{C}$ in degrees Kelvin. There is of course no guarantee that the approximations which lead to Eq. (51) will be accurate for all of the superconducting materials, but the above estimate of $\hat{t}$ is likely to give an order of magnitude value for the freezeout time.

The size of the frozen-out domains will be given by Equation (33) with the exponent $\nu=1 / 2$ :

$$
\xi=\xi_{0} / \sqrt{|\epsilon|} \text {. }
$$

Typical values of $\xi_{0}$ in superconductors are significantly larger than in superfluids (i.e. $\xi \sim 1000 \AA=10^{-5} \mathrm{~cm}$ ) although smaller $\xi_{0}$ can also be found (for example, in high-temperature superconductors). Nevertheless, for the purpose of rough estimates one can evaluate $d$ to be:

$$
d \simeq 10^{-2}\left(\xi_{0} / 1000 \AA\right) \tau_{Q}^{1 / 4}[\mathrm{~cm}]
$$

Hence, the domain sizes are much larger in superconductors than in superfluids. The resulting vortex line density will be therefore correspondingly smaller:

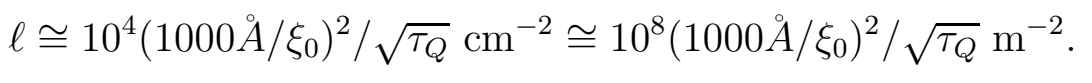

These estimates may still look reasonably hopeful, but it seems unlikely to this author that the values of $\ell$ predicted by Eq. (55) will be easily detectable experimentally: As is the case in the superfluid helium (or in a liquid crystal) domain structure will give rise to the initial network of the flux lines with $\ell$ predicted above, but the evolution shrinking of the loops, straightening of the long string - will quickly lower the value of $\ell$. One could slow down this process by choosing a material with plentiful pinning sites. However, the presence of the inhomogeneities which give rise to pinning may also invalidate some of the arguments we have put forward above by - for example - making the phase transition temperature $T_{C}$ location-dependent. Indeed, this last remark emphasizes one of the great advantages of superfluid helium from the point of view of quench experiments ${ }^{8,9}$ - its homogeneity. 
In view of these considerations, it is clear that the experimental study of flux line creation in rapid phase transitions into the superconductor is bound to be more complicated than in the case of superfluids. These difficulties are mainly of the experimental nature. In particular, both rapid quench and the detection of flux lines appear to be much more difficult to accomplish in superconductors than in superfluid helium. Both of these difficulties may be partially alleviated by using twodimensional samples with the "thin" dimension somewhat larger than $d$, Eq. (54), but much smaller than the other two dimensions. This geometry could help in cooling, and would also allow easier access to the potentially detectable "ends" of flux tubes.

\section{QUENCH IN AN ANNULUS}

Complexity of evolution of the Brownian network of vortex lines makes it worthwhile to discuss a conceptually simpler version of the rapid phase transition into a vacuum with a non-trivial topology of the ground state manifold. With this motivation in mind, we shall consider creation of Bose condensate in superfluid $\mathrm{He}^{4}$ in an annulus. Similar experiments can be also performed in superconducting loops. In the superfluid helium, I shall argue, rapid quench will set up a deficit of the phase $\theta$ which distinguishes between the various degenerate vacua in the broken symmetry state. This phase difference will result in a flow in a random direction, but - for reasonable quench timescales - with a detectable velocity $(\sim 0.1 \mathrm{~cm} / \mathrm{s})$.

Quench into a superconductor will similarly lead to a phase deficit. This phase deficit will translate into a supercurrent, which will trap flux quanta inside the loop. The number of the trapped quanta will, in general, depend on the rate of the phase transition, but may be also influenced by other factors which are normally disregarded in the discussions carried out in the cosmological context such as the inductance and resistivity of the L-R circuit equivalent to the loop. Moreover, geometry of the loop can be made (approximately) one-dimensional, which may (in the appropriate conditions) restore the importance of activation processes. Thus, in one-dimensional superconductors the original version of the Kibble mechanism with its emphasis on thermal activation may be again important (although for reasons which are not expected to be valid in the cosmological context).

\section{Phase Around the Loop: Generating Persistent Superflows With a Quench}

Let us consider an imaginary circular loop of radius $r$ in a bulk superfluid. After the quench the circumference of this loop will intersect approximately:

$$
N=2 \pi r / \xi(\hat{t})=2 \pi r / d
$$

independent domains. Hence, the anticipated phase mismatch will be of the order of;

$$
\Delta \theta \sim \sqrt{N}=\sqrt{\frac{2 \pi}{d}} .
$$


Consequently, the gradient of the average phase will be approximately equal to:

$$
g=\Delta \theta / 2 \pi r=\sqrt{1 / 2 \pi r d} .
$$

In the superfluid, such gradient of the phase implies supercurrent velocity of:

$$
v=(\hbar / m) g=(\hbar / m) /(C d)^{1 / 2},
$$

where $C=2 \pi r$ is the circumference of the loop.

It was already pointed out some time ago ${ }^{4,5}$ that this phase difference (which would decrease in bulk superfluid with the evolution of the vortex line network) can be "frozen out" by performing the quench in an annulus. Moreover, the resulting velocities are measurable $(\sim \mathrm{mm} / \mathrm{s})$ and depend only weakly on the quench timescale;

$$
v \sim \tau_{Q}^{-\frac{\nu}{4}} \approx \tau_{Q}^{-\frac{1}{6}}
$$

where we have used the renormalization group value of $\nu=2 / 3$. The corresponding angular momentum is non-negligible, but there is no paradox, as its origins can be traced to Brownian motion at the "freeze-out time" $\hat{t}^{5}$

The principal advantage of the quench in annular geometry is the time-independence of the effect - persistent supercurrent. In contrast to the vortices created in the bulk superfluid, persistent supercurrents do not decay, or at least do not decay on a rapid timescale on which vortex lines intersect and disappear. Hence, one may have a better chance to obtain an estimate of the frozen-out correlation length. On the other hand, this experiment is significantly more challenging than the bulk version. Among the experimental difficulties one should list the problem of performing the quench in an axially-symmetric fashion (so that the superfluid is not "pushed" in the process) as well as the measurement of the resulting velocity.

There are also theoretical complications: Rapid quench will change the equilibrium correlation length $\xi(T)$ from a large near - $T_{C}$ value to much smaller value far from the $\lambda$-line. This changing value of $\xi(T)$ will be, at some stage, comparable to the small radius $a$ of the torus containing the superfluid. When $a<\xi(T)$, the superfluid is effectively one-dimensional. Thus, vortex lines cannot "fit" within the annular container. Moreover, activation energy required to change the winding number $n_{w}$ defined as;

$$
n_{w}=\frac{\Delta \theta}{2 \pi}
$$

is given by;

$$
\Delta F_{0} \cong \pi a^{2} \xi(T) \Delta F .
$$

When $a<\xi, \Delta F_{0}$ is less than the energy one would normally employ in deriving Ginzburg temperature. For this reason, in the first papers on this subject ${ }^{4,5} \mathrm{I}$ have suggested using annulus with $\xi(\hat{t}) \sim a$ : For $t>\hat{t}(\xi(t)<\xi(\hat{t}))$ coherent fluctuations 
of the volume $\sim a^{3}$ will become unlikely, so that the winding number will be "safe" from thermally activated processes. This conclusion - while essentially valid - ignores creation of small sections of the vortex line inside the annulus in the regime where $a \sim \xi(t)$. Such vortex lines may change the average velocity of the superflow. With time, they may also migrate towards the inner (or outer) wall of the annulus, thus changing the winding number.

In spite of the above concerns, I believe that this "phase around the loop" experiment is very much worth performing: It offers a dramatic demonstration of the "phase freezeout" predicting generation of a significant velocity (and of a measurable angular momentum) as a result of the rapid phase transition. Moreover, the possibility of thermally activated transitions should be regarded not just as a complication, but as an opportunity. Thus, for example, one can contemplate studying of not just quench - generated superflows, but also decay - due to thermally activated processes - of the winding number. In this regime one is probing the interplay between thermal activation and topological stability.

\section{Winding Number in a Superconducting Loop: Quenching out Flux}

For the quench experiments carried out in a superconducting loop the basic scenario of locking out superflow (of Cooper pairs) should be still applicable, although with a few important (and interesting) complications. Let us therefore consider a loop of some radius $r$ with the wire diameter given by $2 a$, where $a \ll r$. We shall suppose that for the quench timescale under consideration the frozen-out correlation length $d$ is at most of the order of $2 a$, so that one has typically no more than one domain across the wire. Rapid transition will then result in a typical phase difference $\Delta \theta$ along the circumference of the wire in accord with Eq. (57). Hence, the resulting winding number $n_{\Phi}$ (and the number of trapped flux quanta) should be of the order:

$$
n_{\Phi}=\frac{\Delta \theta}{2 \pi}=(2 \pi)^{-1} \sqrt{\frac{C}{d}}
$$

For a loop of $r=1 \mathrm{~cm}(C=2 \pi r)$ and the frozen out correlation length of $\sim 10^{-2} \mathrm{~cm}$ (see Eq. (54)) this yields small but easily measurable $n_{\Phi} \sim 3$.

So far we have ignored the role of the gauge field: Our prediction, Eq. (63), is based solely on the fate of the order parameter. Yet, the energy of the trapped flux $E_{\Phi}$ can easily be comparable or even larger than the energy of thermal fluctuations at the temperature at which the phase transition is taking place. Thus:

$$
E_{\Phi}=\Phi^{2} / 2 L=\frac{n_{\Phi}^{2}(h c / 2 e)^{2}}{2 L}=n_{\Phi}^{2} E_{0}
$$

where $L$ is the self-inductance of the loop. It is given approximately by:

$$
L \cong 4 \pi \cdot 10^{-9} r \ln (r / a)[\mathrm{H}]
$$


where $L$ is measured in Henry's and $r$ in centimeters. Energy unit $E_{0}$ in Eq. (64) stands for the energy associated with a single quantum of flux trapped in a loop of self-inductance $L$;

$$
E_{0}=\Phi_{0}^{2} /(2 L) \simeq 2.5 \cdot 10^{-16} r^{-1}[\mathrm{erg}]
$$

where we have taken $r / a=1000$. For comparison, the energy of thermal fluctuations available at the critical temperature is:

$$
E_{T}=\frac{1}{2} k_{B} T_{C} \cong 7 \times 10^{-17} \cdot T_{C}[\mathrm{erg}]
$$

where $T_{C}$ is given in degrees Kelvin. For typical superconductors $T_{C}$ falls in the range of 0.1 to few tens of Kelvins. Thus, even in the relatively high-temperature cases, only a few quanta of the frozen out flux could come directly from thermal fluctuations. Moreover, the value of the inductance (and, consequently, $E_{0}$ ) can be altered by changing the geometry of the loop.

We have therefore three possible sources of the locked-out flux. The first (trivial) is the background flux which is going to determine the average flux through the loop. Dispersion about that average can be either due to the freeze-out of thermal fluctuations of the flux, in which case;

$$
\frac{\delta \Phi_{T}^{2}}{2 L}=\frac{1}{2} k_{B} T_{C}
$$

or it can have the more interesting origin in the freeze-out of the order parameter we have considered above, in which case the dispersion of the flux will be given by Eq. (63), or:

$$
\delta \Phi^{2}=\Phi_{0}^{2} \cdot n_{\Phi}^{2}=\left(\Phi_{0}^{2} /(2 \pi)^{2}\right)(C / d)
$$

It should be (at least in principle) relatively easy to distinguish between these two cases. Freeze-out of the order parameter results in the prediction which is independent of the inductance $L$ of the superconducting loop, but which depends on its circumference $C$. Thus, one can tell the difference between the two cases by deforming the loop (i.e., by coiling it up) which will change $L$, but leave $C$ unaltered. Moreover, freeze-out of the order parameter should result in slow variations of the flux:

$$
n_{\Phi} \sim \tau_{Q}^{-1 / 8}
$$

with the quench timescale. In addition to those signatures one can of course compare the absolute value of the predicted flux variations.

It should be emphasized that the question which is being posed here is a profound one: We are trying to determine whether it is the gauge field or the order parameter which controls the final frozen-out winding number. In the discussions carried out in the cosmological context the usual assumption associates topological defect formation 
with the correlation length of the order parameter. If this were indeed the case, then the estimate given by Eq. (69) should prevail. There are however circumstances in which thermal fluctuations of the gauge field (rather than of the order parameter) may emerge from the experiments. The first one is relatively trivial: Let us suppose that the phase transition does not happen simultaneously around the loop, but there is one spot which becomes superconducting last. Then, for a while, the order parameter along the superconducting section of the loop (and the flux associated with it) will be in contact with a thermal reservoir at (approximately) $T_{C}$. The whole of the loop is not superconducting. Therefore, the flux inside it is still able to vary, presumably on a timescale associated with the inductance:

$$
\tau_{R L}=L / R
$$

where $R$ is the resistance of the normal section of the loop. Consequently, as the still normal section of the loop becomes superconducting (thus fixing the value of the flux) the order parameter coupled with the flux of the magnetic field continues to be driven by thermal fluctuations.

In this limit, quench becomes reminiscent of the experiment carried out by Tate et al. ${ }^{15}$ They have repeatedly heated a small section of superconducting niobium ring. Upon cooling, the ring had contained a random number of quanta with a dispersion corresponding to $6.78^{\circ} \mathrm{K}$ which is somewhat less than the critical temperature of pure niobium (which is $9.17^{\circ} \mathrm{K}$ ). It is clear that in this limit (when the loop is kept open for periods of time long compared to all other physically relevant timescale) the number of quanta eventually locked out will be determined by thermal fluctuations.

It should be noted that the geometry of the loop which is undergoing the quench is different from the one relevant in the cosmological context. The key consequence of this difference has to do with the fact that inside the loop gauge field remains massless even after the phase transition. This is clearly not going to be so in the early universe, where the critical temperature is reached more or less simultaneously throughout the volume. Nevertheless, experiments proposed here should allow one to shed a new light on the relative roles of the gauge field and of the order parameter in general. This point is of significant interest, and related questions about the cosmological phase transitions have also been raised. ${ }^{16}$

The crucial point which is relevant for the loop geometry in this context concerns the nature and the rates of the processes which could alter the winding number between the instant of its creation $\hat{t}$ and the later time when it can be measured. Two of them can be readily identified: (i) Ginzburg - like activation which acts locally (that is, within the correlation length $\xi$ ) and on the order parameter, and (ii) Transitions involving the whole loop and mediated by the (still massless) gauge field trapped inside. Both of these processes will be effective only near the critical temperature. Thus, once the relatively narrow (few milikelvin, at the most) danger 
zone is traversed in course of the quench, the number of flux quanta shall be fixed and can be measured at leisure.

The role of the activation processes in thin wires (that is, in the case when the radius of the wire is smaller then the correlation length) has been studied (see chapter 7 of Ref. 12). It is determined by the free energy increment needed to "flip" the order parameter over the potential barrier in a small section $(\sim \xi)$ of the loop. The exact expression turns out to be given by:

$$
\Delta F_{0}=\frac{4 \sqrt{2}}{3} \frac{\alpha^{2}}{\beta} A \xi
$$

Above, $A$ is the cross section of the wire.

Theory and experiment both agree that unless $A$ is very small $\left(A \sim 10^{-9} \mathrm{~cm}^{2}\right)$ so that the volume of the section of the wire over which the transition occurs is much less than $\xi^{3}$, such transitions cannot be expected to take place within less than $\sim 10^{-3}{ }^{\circ} \mathrm{K}$ of the critical temperature. As the temperature - dependent terms in $\Delta F_{0}$ change with $|\epsilon|^{3 / 2}$, it should be possible to select parameters so as to assure that the Ginzburg - like process considered here will not change the winding number. For example, the rate can be lowered by increasing the cross section of the wire to $A \sim 10^{-4} \mathrm{~cm}^{2}\left(a \sim 10^{-2} \mathrm{~cm}\right)$. In effect, as was the case for the quench in the "loop" of the superfluid helium, one would want to select $a$ of the order of $d=\xi(\hat{t})$ to keep the problem effectively one - dimensional, while, at the same time, making activation energetically as expensive as in the bulk.

The second process which can influence the number of captured flux quanta has a global nature (or, at the very least, its rate does not depend on $\xi$ ) and is mediated by the magnetic field trapped inside the loop. The activation energy associated with it can be estimated by computing the kinetic energy of the Cooper pairs in the case when the enclosed flux does not correspond to an integer number of flux quanta.

Just below the phase transition London penetration depth $\lambda$ can be large in comparison with the diameter of the wire, $\lambda>2 a$. Then the current density in the wire will be (approximately) independent of location (that is, same on the inside and on the outside of the loop). The total energy (corresponding to a certain current density) will comprise of two contributions: The energy of the magnetic flux (given by Eq. (64)) and the kinetic energy of the charge carriers. For an arbitrary flux one may choose $n_{\Phi}$ so that the residual wave vector:

$$
q=(2 \pi / C)\left(n_{\Phi}-\Phi / \Phi_{0}\right)
$$

is in the interval $-\pi<q C<\pi$. This corresponds to the velocity:

$$
v=\hbar q / m_{C}
$$


where $m_{C}$ is mass of the Cooper pair. The total energy will be then given by:

$$
E=\Phi^{2} /(2 L)+N_{C} m_{C} v^{2} / 2
$$

where $N_{C}$ is the total number of the Cooper pairs in the loop. $N_{C}$ can be (in our case) obtained form the density of the pairs $n_{C}$, (which is given by the square of the order parameter):

$$
N_{C}=C \pi a^{2} n_{C}
$$

Quantization of the flux is the consequence of the existence of the local minima of $E$ as a function of $\Phi$. Using Eq. (73) - (76) it is straightforward to show that $E$ is minimized when;

$$
\Phi=n_{\Phi} \Phi_{0} /\left(1+E_{0} / E_{K}\right)
$$

where $E_{0}$ corresponds to the energy of a single quantum of flux, Eq. (66), and $E_{K}$ is the kinetic energy due to the velocity resulting from the phase difference of $2 \pi$ over the circumference $C$ and is given by:

$$
E_{K}=\frac{1}{2} N_{C} m_{C}\left[\left(\hbar / m_{C}\right)(2 \pi / C)\right]^{2} .
$$

Consequently, in equilibrium the flux in a loop of a given self-inductance $L$ and circumference $C$ will be quantized in the units of ${ }^{17-19}$ :

$$
\tilde{\Phi}_{0}=\Phi_{0} /\left(1+E_{0} / E_{K}\right)
$$

Moreover, quantization is not "absolute": Transitions between the different minima of the total energy - corresponding to different integer values of flux - are guarded against by the potential barrier with the height:

$$
E_{B} \cong E_{K} / 2
$$

At $t=\hat{t}$ this energy is generally smaller than the free energy $\Delta F_{0}$, Eq. (72), and may be of the order of $k_{B} T_{C}$. However, changes of flux cannot take place on a timescale smaller than $\tau_{R L}$, Eq. (71). Moreover, near $T_{C}$ the resistance $R$ of the loop is expected to rapidly decrease, and $\tau_{R L}$ will increase.

It is difficult to tell whether these processes will allow the flux to acquire its order parameter mandated value, Eq. (63), or if the trapped gauge field will assume value closer to the thermal prediction, Eq. (68). With Pablo Laguna, we are presently carrying out a numerical study of rapid quenches in (quasi-) one-dimensional loop configurations to clarify some of the relevant issues ${ }^{20}$. The above concerns notwithstanding, it may turn out that the most challenging difficulties in carrying out actual experiments may be of more "mundane" nature, and may involve making sure that the critical temperature is reached (nearly) simultaneously around the loop, that the loop is screened from outside magnetic fields, etc. 
One could also contemplate artificially controlling the number of the independent pieces of the superconducting order parameter (instead of relying on $\xi$ ). This could be achieved, for example, by heating up the loop in many places, so as to break up the superconducting loop into many pieces, (say, $N$ ), which should result in a phase difference $\sim \sqrt{N}$ (in addition to the fluxoid due to the thermal activation of the field, Eq. (68)), and a corresponding winding number after the reconnection. Many variants of such experiments are possible. We shall not analyze them here further.

\section{COSMOLOGICAL IMPLICATIONS}

Throughout much of this paper we have focused on condensed matter systems, or, to be more specific, on condensed matter analogues of the cosmological "quench". The central lesson of our discussion was a revision of the original mechanism for defect formation in the course of second order phase transitions involving a non-conserved order parameter ${ }^{2}$. We have concluded that the initial density of the topological defects will be set by the correlation length at the freezeout instant $\hat{t}$ - that is, at the moment when the relaxation timescale of the order parameter will be comparable to the time from when the critical temperature $T_{C}$ is attained. ${ }^{4,5,13}$ At that instant, as a consequence of critical slowing down, perturbations of the order parameter become so sluggish that they effectively cease to evolve, so that in the time interval $(-\hat{t}, \hat{t})$ the order parameter cannot adjust its correlation length to the values of the thermodynamic parameters which are changing as a result of the quench. When, below $T_{C}$, and for $t>\hat{t}$, the dynamics will "restart", it will be too late to get rid of the defects, which have been by then "set in concrete" (or, rather, in the topologically stable configurations of the order parameter).

Experimental results obtained in the superfluid experiment by the Lancaster group $^{8,9}$ demonstrate that this argument is unaffected by the thermal activation process invoked in the original discussion by Kibble ${ }^{2}$. Ginzburg temperature - that is, the temperature at which vacuum can be thermally "flipped" over the potential barrier in regions of size $\xi$ - is far below the critical temperature $T_{\lambda}$ in the superfluid helium case $\left(T_{\lambda}-T_{G} \simeq 0.5^{\circ} \mathrm{K}\right)$. In spite of that, there is no evidence for copious thermal creation of vortex lines. We expect that the same situation will prevail in superconductors, where $T_{G}$ is very close to $T_{C}$, even though the freezeout temperature will be now typically lower than $T_{G}$. This is not unexpected - topological defects created by the activation process are very small $(\sim \xi)$. Therefore, topology does not yet stabilize them: Decay of a doughnut - shaped string loop can take place on a relaxation timescale, as it involves getting rid of the "hole" in the center of the doughnut, and is obviously energetically favored.

In superconductors, where $T_{G}$ can be expected to be above the freezeout tempera-

ture reached during the quench at $\hat{t}$, that is, within the "time out" interval $(-\hat{t}, \hat{t})$, this expectation is even easier to justify: The order parameter is simply too sluggish to do 
anything between $T_{C}$ and $T_{G}$. However, for superconductors a different timescale and a different lengthscale are also relevant. In addition to the order parameter (which is still expected to play a pivotal role there is also the gauge field, which may be quite important in the loop geometry, but may have some significance also in the bulk ${ }^{16}$. It is hoped that the experiments suggested above can be carried out and that they will shed as much light on the dynamics of phase transitions with the local gauge as the Lancaster superfluid experiment already did for the case of the global gauge ${ }^{8,9}$.

Last but not least, let us briefly consider implications of our observations concerning the relative roles of the freezeout instant and Ginzburg temperature in the cosmological context. To carry out this discussion, we shall adopt the usual high energy / cosmology unit system with $\hbar=c=k_{B}=1$, and focus on the order parameter of the effective field theory described by the Lagrangian given either by Eq. (21) or (24). The principal difference with superfluids and superconductors is due to the fact that the corresponding equation of motion has a second time derivative on the left hand side (in contrast to the first derivative relevant for the "nonrelativistic" condensed matter cases). Hence, the relaxation timescale of the order parameter will be given by:

$$
\tau=1 / \sqrt{\alpha}=\tau_{0} / \sqrt{|\epsilon|} .
$$

That is, critical slowing down sets in with the inverse of the square root of the relative temperature, rather than with $1 /|\epsilon|$, as was the case for Eq. (32). One immediate consequence of this difference is that the corresponding characteristic velocity defined by $\xi / \tau$ with which perturbations of the order parameter can spread will be finite and relatively unchanged in the vicinity of $T_{C}$. By contrast, the second sound velocity in the superfluid decreases as $|\epsilon|^{1-\nu}$ in the vicinity of $T_{C}$.

The cosmological version of Eq. (35) for the freezeout instant can be now written:

$$
\tau_{0} / \sqrt{|\epsilon(\hat{t})|}=\hat{t}
$$

which, with the usual definition of the quench timescale $\left(\epsilon=t / \tau_{Q}\right.$, Eq. (34)) yields:

$$
\hat{t}=\tau_{0}^{\frac{2}{3}} \tau_{Q}^{\frac{1}{3}}
$$

This is in contrast to the previously derived and more symmetric $\hat{t}=\sqrt{\tau_{0} \tau_{Q}}$, Eq. (37), which is valid for the superfluid and for the superconductor case, when $\tau \sim 1 /|\epsilon|$.

We are now ready to compare and contrast prediction for the freezeout scenario with the Ginzburg activation mechanism. In the radiation - dominated era the temperature $T$ and the time $t$ (which is measured form the beginning of time - from the Big Bang) are related with a simple equality:

$$
T^{2} t=\Gamma M_{P l}
$$

where $M_{P l}$ is the Planck mass, while;

$$
\Gamma=(1 / 4 \pi) \sqrt{45 /(\pi \varsigma)}
$$


and $\varsigma$ is the effective number of different spin states of relativistic particles. Using this and defining $\tau_{Q}=1 / \dot{\epsilon}$ at the instant when the critical temperature is reached we get:

$$
\tau_{Q}=2 \Gamma M_{P l} / T_{C}^{2}
$$

We can also estimate, by using a (weak coupling) approximate equality;

$$
T_{C}=\sqrt{\alpha(T=0) / \beta}=\sqrt{\alpha^{\prime} / \beta}
$$

so that:

$$
\tau_{0}=1 /\left(\sqrt{\beta} T_{C}\right)
$$

Consequently,

$$
\hat{t}=\left(\beta^{\frac{1}{3}} T_{C}\right)^{-1}\left(2 \Gamma M_{P l} / T_{C}\right)^{\frac{1}{3}}
$$

and;

$$
\epsilon(\hat{t})=\left(T_{C} /\left(2 \Gamma M_{P l}\right)\right)^{\frac{2}{3}} / \beta^{\frac{1}{3}}
$$

By contrast, the relative temperature corresponding to the Ginzburg condition, Eq. (45), is simply given by:

$$
\epsilon_{G}=2 \beta
$$

Here, we have again used the approximate relation, Eq. (88), as well as Eq. (9) of Ref. 2.

Thus, it appears that the initial density of defects expected on the basis of the freezeout scenario (which seems to have been borne out in the superfluid helium Lancaster experiment ${ }^{8,9}$ ) is quite different from the one anticipated on the basis of the activation (Ginzburg) version of the original Kibble mechanism ${ }^{2}$. This difference will be especially pronounced in the phase transitions occurring at lower energies, where $T_{C} / M_{P l} \ll 1$. Examination of the cosmological consequences of this result is beyond the scope of this paper.

\section{ACKNOWLEDGEMENTS}

Discussions with Andreas Albrecht, Robert Brandenberger, Ike Chuang, Anne Davis, Alasdair Gill, Nigel Goldenfeld, Tom Kibble, Bernard Yurke, Alex Vilenkin, Wojtek Zakrzewski and other participants of the Topological Defects Programme are gratefully acknowledged. I have especially benefited from the interactions with Peter McClintock and Richard Lee concerning the superfluid helium experiment they (and their colleagues) have carried out. Isaac Newton Institute provided a partial support and a stimulating environment for much of the research reported here. Discussion of Section 4. of this paper has been influenced by the collaboration with Pablo Laguna aimed at the numerical study of the evolution of the order parameter, and carried out with the support of NASA HPCC "Grand Challenge" initiative. 


\section{REFERENCES}

1. Ya. B. Zeldovich, I. Yu. Kobzarev, and L. B. Okun, Cosmological consequences of a spontaneous breakdown of discrete symmetry, Sov. Phys. JETP, 40:1-5 (1975).

2. T. W. B. Kibble, Topology of cosmic domains and strings, J. Phys. A: Math. Gen. 9:1387-1398 (1976); also, T. W. B. Kibble, in this volume.

3. A. Vilenkin and E. P. S. Shellard, Cosmic Strings and other Topological Defects (Cambridge Univ. Press, Cambridge, 1994).

4. W. H. Zurek, p. 479 in Proc. DPF Meeting of APS, T. Goldman and M. M. Nieto, eds., (World Scientific, Singapore, 1985); W. H. Zurek, Experimental cosmology: Strings in superfluid helium, Los Alamos preprint LAUR 84-3818 (1984).

5. W. H. Zurek, Cosmological experiments in superfluid helium? Nature 317: 505-507 (1985).

6. I. Chuang, R. Durrer, N. Turok, and B. Yurke, Cosmology in the laboratory: Defect dynamics in liquid crystals, Science 251:1336-1338 (1991).

7. B. Yurke, Coarsening dynamics in liquid crystal systems, this volume.

8. P. C. Hendry, N. S. Lawson, R. A. M. Lee, P. V. E. McClintock, and C. H. D. Williams, Generation of defects in superfluid $\mathrm{He}^{4}$ as an analogue of the formation of cosmic strings, Nature 368:315-317 (1994).

9. P. C. Hendry, N. S. Lawson, R. A. M. Lee, P. V. E. McClintock, and C. H. D. Williams, Cosmological experiments in $\mathrm{He}^{4}-$ problems and prospects, this volume.

10. M. J. Bowick, L. Chandar, E. A. Schiff, and A. M. Srivastava, The cosmological Kibble mechanism in the laboratory: String formation in liquid crystals, Science 263:943-945 (1994).

11. D. R. Tilley and J. Tilley, Superfluidity and Superconductivity, second edition (Hilger, Boston, 1986).

12. M. Tinkham, Introduction to Superconductivity, (McGraw-Hill, New York, 1975).

13. W. H. Zurek, Cosmic strings in laboratory superfluids and topological remnants of other phase transitions, Acta Physica Polonica 24:1301-1311 (1993). 
14. E. A. Lynton, Superconductivity, third edition, (Menuthen, London, 1969).

15. J. Tate, B. Cabrera, and S. B. Felch, Novel noise thermometer for measuring the local critical temperature of a superconducting ring, in LT-17, U. Eckern, A. Schmid, W. Weber, and H. Wühl, eds. (Elsevier, Amsterdam, 1984).

16. S. Rudaz and A. M. Srivastava, Mod. Phys. Lett. A8:1443-1445 (1993).

17. J. M. Blatt, Persistent ring currents in an ideal Bose gas, Phys. Rev. Lett. 7:82-83 (1961).

18. J. Bardeen, Quantization of flux in superconducting cylinder, Phys. Rev. Lett. 7:162-163 (1961).

19. J. B. Keller and B. Zumino, Quantization of fluxoid in superconductivity, Phys. Rev. Lett. 7:164-165 (1961).

20. P. Laguna and W. H. Zurek, work in progress. 